\title{
Articular Tuberculosis Affecting the Distal Humerus
}

\author{
Aryan Vahedi-Faridi, MD, MS' - Kevin Govani' - Michael Ebisi² - Sumera Khan ${ }^{3}$ Pranav Patel, MD ${ }^{4}$
}

In December 2018, a 27-year-old man who had recently emigrated to the United States from India had struck his right elbow into a door. Over the following weeks, he had had experienced progressive pain and swelling in the joint. He had had sought treatment at a hospital, and traumatic bursitis had been diagnosed. At that time, his treatment had had included casting of the joint and physical therapy. However, the patient had not adhered to follow-up management, and therefore, his symptoms had continued to worsen. He then sought treatment at our clinic in July 2020.

\section{History}

His medical history was unremarkable. His social history was negative for alcohol, tobacco, or intravenous drug use. Notably, he had received a scheduled Bacille Calmette-Guérin (BCG) vaccine in India.

\section{Physical examination}

Edema of the right elbow without erythema was noted. Flexion, pronation, and supination were severely limited because of contracture of the forearm. The patient reported significant pain with active and free range of motion. Lymphadenopathy was absent. There were no other remarkable findings.

Results of laboratory tests were within the normal ranges for white blood cell count, erythrocyte sedimentation rate, C-reactive protein, rheumatoid factor, and uric acid. Results of a QuantiFERON-TB-Gold (QFT) blood test were positive. Given these results, further inquiry into the injury was made, but the patient denied any history of pulmonary tuberculosis (TB) symptoms (ie, fever, night sweats, bloody sputum) and reported no ill contacts.

An orthopedist consultation led to repeat diagnosis of traumatic arthritis. A radiograph of the patient's elbow showed an osteopenic lesion in the

\section{AFFILIATIONS:}

'Saint James School of Medicine, Anguilla

${ }^{2}$ Avalon University School of Medicine, Curacao, Curaçao

${ }^{3}$ Windsor University School of Medicine, Kitts \& Nevis

${ }^{4}$ Department of Internal Medicine, Palos Medical Care, Palos Heights, Illinois

\section{CITATION:}

Vahedi-Faridi A, Govani K, Ebisi M, Khan S, Patel P. Articular tuberculosis affecting the distal humerus. Consultant. Published online June 1, 2021. doi:10.25270/con.2021.06.00001

Received December 18, 2020. Accepted January 29, 2021.

\section{DISCLOSURES:}

The authors report no relevant financial relationships.

\section{CORRESPONDENCE:}

Aryan Vahedi-Faridi, MD, MS, Saint James School of Medicine, Albert Lake Drive The Quarter, A-1 2640, 2640, Anguilla (afaridi@mail.sjsm.org)

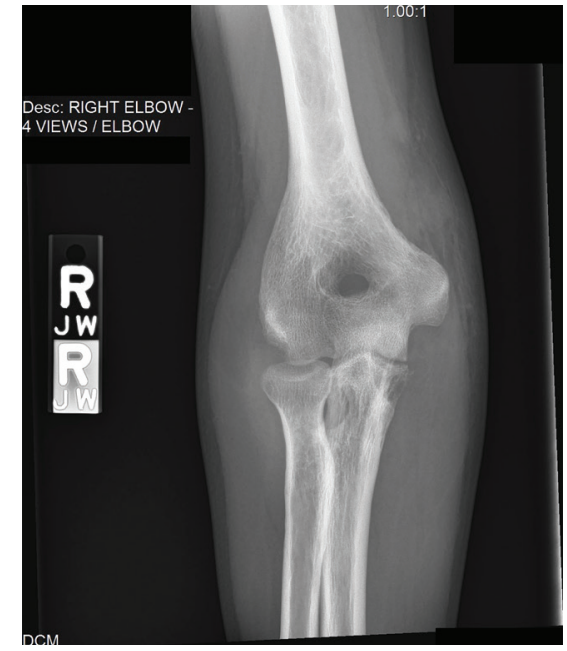

Figure 1. The radiograph of the patient's right elbow demonstrated significant osseous erosion and osteopenia in the distal humerus.

distal right humerus (Figure 1). A computed tomography scan demonstrated additional effusions, intraarticular adhesions, and erosions of the capitellar and ulnohumeral articulations. Synovial fluid aspirated from the joint did not produce findings consistent with septic arthritis.

In September 2020, surgical debridement with contracture release was performed. Specimens collected during the procedure demonstrated positive histopathology with reactive bone and granulomatous findings (Figure 2). Results of an acid-fast stain of collected samples were negative. However, a mycobacterial culture with DNA probe returned positive results. Susceptibility was also tested, which demonstrated unsuppressed results for rifampin, isoniazid, pyrazinamide, and ethambutol.

A consultation with an infectious disease specialist noted that the patient's imaging findings, histopathology, 


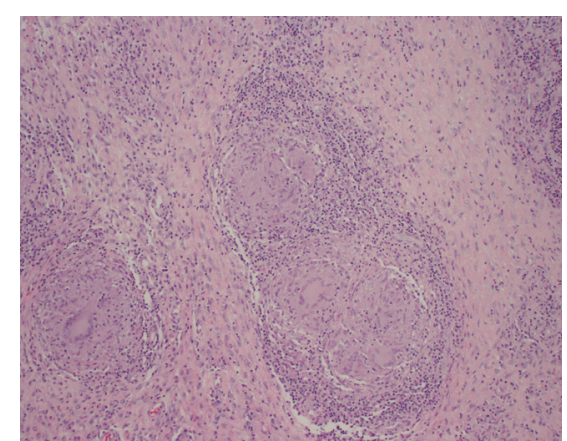

Figure 2. Histopathology of an elbow bone specimen collected during surgical debridement demonstrated reactive bone and granulomatous findings.

culture, and positive QFT test could indicate articular TB. The patient was started on first-line anti-TB therapy, which included rifampin, isoniazid, pyrazinamide, and ethambutol for 2 months followed by rifampin and isoniazid for 4 to 8 months. Physical therapy was also prescribed.

\section{Discussion}

There are few reports of articular TB affecting the distal humerus available in the current literature. At our clinic, a 28-year-old man presented with a 1-year history of progressive right elbow pain and swelling and no constitutional symptoms. His history was significant for trauma and exposure to a TB-endemic region. Results of a QFT test were positive, and elbow imaging demonstrated erosion, osteopenia, and effusion. Interestingly, his chest radiograph was unremarkable for pulmonary TB. Bone tissue samples collected during surgical debridement were important for diagnosis and yielded a culture positive for TB and granuloma- tous findings on histopathology.

We suspect that our patient had developed skeletal TB after hematogenous spread from a primary source or by reactivation of a dormant lesion. The patient had experienced insidious development of pain and swelling in the joint after mild trauma, which is consistent with osseous granular type osteoarticular TB. ${ }^{1}$ Delayed diagnosis after symptom onset likely allowed the lesion to grow and worsen the patient's symptoms. Since beginning treatment with surgery and long-term antibiotics, the patient has reported improved range of motion and pain at each of his follow-up visits. Definitive therapy via elbow joint replacement has been considered, should his condition continue to worsen.

Diagnosis of articular TB can be difficult because of presentation overlap with other, more common forms of arthritis (ie, septic arthritis)..$^{2-4}$ At present, diagnosis may take between 5 to 47 months. ${ }^{2}$ In this adult from India, assessment of his risk factors was important to establish a diagnosis. Taking a comprehensive history and screening for risk factors are needed to make an early diagnosis of articular TB. Interestingly, our patient's prior immunization with the BCG vaccine did not prevent the development of articular TB.

This case serves to broaden the current clinical picture of articular TB affecting the distal humerus. Presentation, diagnostic imaging, laboratory workup, and histopathology from this case should improve the current archive and clinical detection of this rare disease in the future.

\section{Patient outcome}

The patient's range of motion has improved with up to $50^{\circ}$ of supination, $75^{\circ}$ of pronation, $45^{\circ}$ of extension, and $90^{\circ}$ of active flexion at his most recent visit, 8 months after the procedure. His liver enzyme profile was within normal limits, with no elevations related to isoniazid treatment. Additional screening of his HbA1c level and HIV status, as well as a repeat chest radiography scan, were performed, results of which were unremarkable. The patient is currently in the continuation phase of his antibiotic therapy and has maintained adherence to treatment. Because of his reported improvement in symptoms, joint replacement has not been pursued.

\section{REFERENCES}

1. Husain N, Shukla S. Pathology relevant to the radiologist. In: Gupta RK, Sharma R, eds. Imaging in Tuberculosis: Clinicopathological Correlation. 1st ed. Jaypee Brothers Medical Pub; 2019: 25-39.

2. Liao Q, Shepherd JG, Hasnie S. Mycobacterium tuberculosis of the elbow joint. BMJ Case Rep. 2017;2017:bcr2017222735. https://doi.org/10.1136/bcr-2017-222735

3. Thimmaiah VT, Deepashree. Unusual presentation of tuberculosis of elbow joint: a case report. Res Rev J Med Health Sci. 2013;2:17-20. https://www.rroij.com/ open-access/unusual-presentation-of-tuberculosis-of-elbow-joint-a-case-report. php?aid $=34684$

4. Chen WS, Wang CJ, Eng HL. Tuberculous arthritis of the elbow. Int Orthop. 1997;21(6):367-370. https://doi.org/10.1007/ s002640050187 